\title{
Serotypes and genotypes of $S$. Pneumoniae isolates from adult invasive disease in Spain: A 5-year prospective surveillance after pediatric PCV13 licensure. The ODIN study
}

\author{
Asunción Fenoll $^{\mathrm{a}, 1}$, Carmen Ardanuy b,c,*,1, Josefina Liñares ${ }^{\mathrm{b}, \mathrm{c}}$, Emilia Cercenado ${ }^{\mathrm{c}, \mathrm{d}}$, Francesc Marco ${ }^{\mathrm{e}, \mathrm{f}}$, \\ Ana Fleites ${ }^{g}$, María Rodríguez-Mayo ${ }^{\mathrm{h}}$, Jose-Luis López-Hontangas ${ }^{\mathrm{i}}$, Begoña Palop ${ }^{\mathrm{j}}$, Ana-Isabel Aller ${ }^{\mathrm{k}}$, \\ Buenaventura Buendía ${ }^{1}$, Cristina Méndez ${ }^{m}$, Isabel Cifuentes ${ }^{m}$, the ODIN Study Group
}

a Spanish Reference Laboratory for Pneumococci, Centro Nacional de Microbiología, Madrid, Spain

${ }^{\mathrm{b}}$ Microbiology Department, Hospital Universitari de Bellvitge-IDIBELL, Barcelona, Spain

${ }^{\mathrm{C}}$ CIBER de Enfermedades Respiratorias (CIBERES), Madrid, Spain

${ }^{\mathrm{d}}$ Microbiology and Infectious Diseases Department, Hospital General Universitario Gregorio Marañón, Madrid, Spain

${ }^{\mathrm{e}}$ Microbiology Department, Hospital Clínic, Barcelona, Spain

f ISGlobal, Barcelona Ctr. Int. Health Res., Hospital Clínic - Universitat de Barcelona, Barcelona, Spain

${ }^{\mathrm{g}}$ Microbiology Department, Hospital Universitario Central de Asturias, Oviedo, Spain

${ }^{\mathrm{h}}$ Microbiology Department, Complejo Hospitalario Universitario A Coruña, A Coruña, Spain

${ }^{\mathrm{i}}$ Microbiology Department, Hospital Universitario y Politécnico La Fe, Valencia, Spain

${ }^{\mathrm{j}}$ Microbiology Department, Hospital Regional Universitario Carlos Haya, Malaga, Spain

${ }^{\mathrm{k}}$ Microbiology Department, H. Nuestra Señora de Valme, Sevilla, Spain

${ }^{1}$ Microbiology Department, Hospital Universitario de La Princesa, Madrid, Spain

${ }^{\mathrm{m}}$ Medical Department, Pfizer, Madrid, Spain

\section{A R T I C L E I N F O}

\section{Article history:}

Received 17 July 2018

Received in revised form 15 October 2018

Accepted 31 October 2018

Available online $\mathrm{xxxx}$

\section{Keywords:}

Streptococcus pneumoniae

PCV13

Serotype

Clonal complex

Invasive pneumococcal disease

\begin{abstract}
A B S T R A C T
Serotypes/genotypes causing invasive pneumococcal disease (IPD) in adults are determined by vaccination strategies. The aim of this study was to assess the epidemiology of IPD in adults ( $\geq 18$ years) after PCV13 introduction for children: serotypes, clonal complexes, antibiotic non-susceptibility and clinical presentations.

We performed a prospective, clinical surveillance of hospitalized culture-confirmed IPDs in adults in nine Spanish hospitals (August 2010-June 2015). A total of 1087 culture-confirmed IPD episodes were included, of which 772 (71.0\%) had bacteremic pneumonia (401 complicated/371 uncomplicated pneumonia), 122 (11.2\%) meningitis, 102 (9.4\%) non-focal bacteremia, 34 (3.1\%) peritonitis and 57 (5.3\%) others. The most common serotypes were: 3 (12.7\%), 19A (8.5\%), 8 (7.7\%), $7 \mathrm{~F}(6.3 \%), 1(4.2 \%), 6 \mathrm{C}$ (4.2\%), 11A (4.2\%), 22F (4.2\%) and 14 (4.0\%). Vaccine types (PCV13 $+6 \mathrm{C}$ ) caused $49.8 \%$ of IPD episodes, with a significant decrease over the 5 -year period, and significant decreases in serotypes $6 \mathrm{C}$ and $7 \mathrm{~F}$. The most common genotypes were: CC180 (8.4\%), CC191 (6.0\%), and CC53 (5.0\%).

Vaccine types caused 53.9\% (414/768) pneumonia episodes and 58.9\% (235/399) complicated pneumonia, 53.4\% IPD in adults <50 years (143/268), and 54.7\% IPD in immunocompetent patients (337/616). Overall non-susceptibility was $25.9 \%$ to penicillin (1.1\% for parenteral criteria), $24.9 \%$ to erythromycin and $2.7 \%$ to levofloxacin.

Conclusions: Although the percentage of vaccine-types causing IPDs in adults significantly decreased, it remained high. Associations of vaccine types with pneumonia (with complicated pneumonia for specific serotypes), and immunocompetent patients point to the burden of IPD caused by PCV13 serotypes.
\end{abstract}

(c) 2018 Elsevier Ltd. All rights reserved.

\footnotetext{
* Corresponding author at: Microbiology Department, Hospital Universitari de Bellvitge-IDIBELL, C/FeixaLlargasn, 08907, L'Hospitalet de Llobregat, Barcelona, Spain.

E-mail address: c.ardanuy@bellvitgehospital.cat (C. Ardanuy).

$1 \mathrm{AF}$ and CA contributed equally to this work and to this manuscript.
}

\section{Introduction}

Streptococcus pneumoniae is a major human pathogen causing pneumonia, meningitis and other invasive diseases. The capsular polysaccharide is the main pneumococcal virulence factor that 
has been associated with differences in the ability of $S$. pneumoniae strains to colonize the nasopharynx and cause invasive disease, as well as variations in disease manifestation, population groups affected, antimicrobial resistance and geographical distribution [1]. Moreover, due to the plasticity of its genome, S. pneumoniae is able to respond to human interventions such as antibiotic treatment and vaccination for disease prevention. Serotype replacement can occur through expansion of existing serotypes/clones, emergence of new clones expressing non-vaccine serotypes or capsular switching [2]. This opens the question as to whether the genetic background (defined by lineages) has a role in clinical/epidemiological characteristics classically associated with capsules. Molecular typing, in addition to capsular serotyping, might provide valuable information on evolutionary changes and subsequent clinical consequences.

Soon after the introduction of the 7-valent pneumococcal conjugate vaccine (PCV7) and subsequent 13-valent PCV (PCV13) in childhood immunization programs, significant and progressive reductions were reported in the incidence of invasive pneumococcal disease (IPD) in children and adults [3-6], due, in the latter, to the herd effect [7-9]. Nevertheless, the higher incidence rates of IPD remain in older adults and children younger than 5 .

Geographic serotype distribution is variable and partly conditioned by antimicrobial consumption and vaccine strategies. In Spain, PCV13 was introduced in June 2010 for immunization of healthy children but, in contrast to most European countries, it was not included into the universal immunization programm
(NIP) and it was only available privately to parents until 20152016, except in two regions, Madrid (June 2010) and Galicia (January 2011) (Fig. 1).

The aim of this study was to perform an in-depth analysis of the serotype and genotype epidemiology of IPD in adults in Spain. To that end, we conducted a multicenter study to analyze the current serotype and genotype distributions, as well as the antibiotic susceptibility of invasive pneumococci. We also analyzed the relationship between serotypes and clones by clinical presentation of IPD.

\section{Materials and methods}

This is a prospective multicenter laboratory-based study performed in nine teaching hospitals of six Autonomous Regions in Spain (August 2010-June 2015). Adult patients ( $\geq 18$ years) with IPD episodes were eligible to participate if they were hospitalized at the study site for at least $24 \mathrm{~h}$. The study protocol was approved by the corresponding local ethics committee, and all patients provided written informed consent. Clinical follow-up was performed until discharge (or up to $\approx 90$ days after if incomplete resolution at discharge) or death.

IPD was defined as the isolation of $S$. pneumoniae in normally sterile fluids (blood, cerebrospinal fluid, pleural fluid, ascitic fluid and others) from a patient with clinical signs/symptoms of infection. The patient's age, immune status, and major clinical presentation (uncomplicated pneumonia, complicated pneumonia, meningitis, non-focal bacteremia, peritonitis and others) were

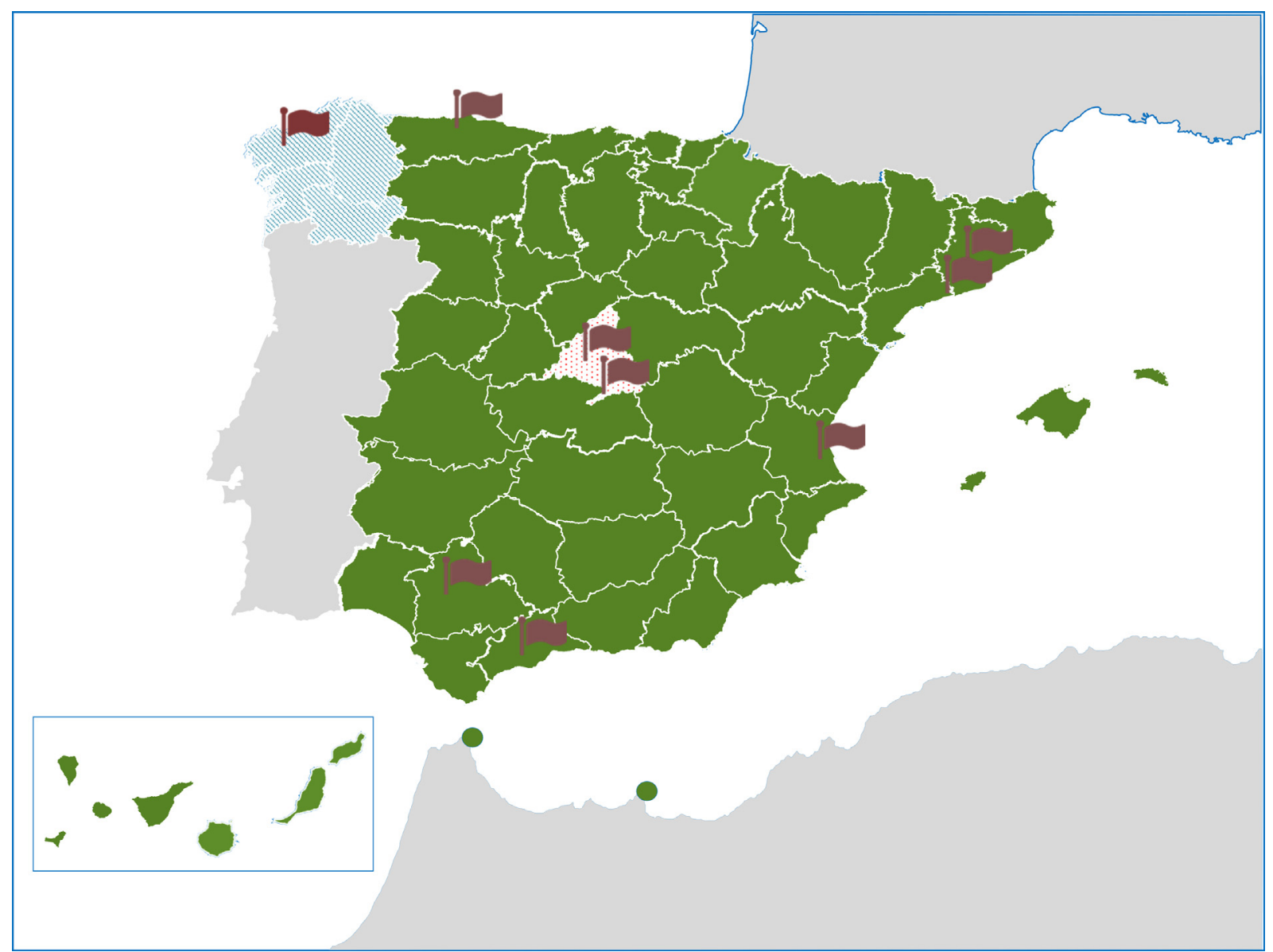

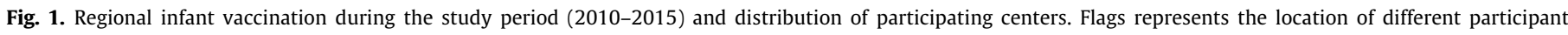

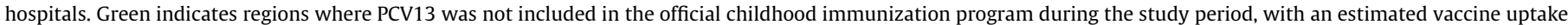

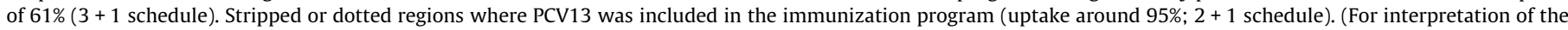
references to color in this figure legend, the reader is referred to the web version of this article.) 
recorded. Pneumonia with empyema, parapneumonic pleural effusion or multilobar infiltration was considered as complicated. As per the Advisory Committee on Immunization Practices (ACIP), patients were considered immunocompromised when cancer, HIV infection, chemotherapy, immunosuppressive treatment, chronic renal disease, nephrotic syndrome, or other causes of immunosuppression or immunodeficiency were present [10].

Isolates were sent to the Spanish Reference Laboratory for Pneumococci and serotyped using the Quellung reaction/dot blot assay [11]. Only one isolate per episode was considered. Molecular typing was performed by pulsed-field gel electrophoresis (PFGE). Genomic DNA embedded in agarose plugs was restricted with SmaI (New England BioLabs), and fragments were separated by PFGE [12]. PFGE patterns were compared with representative international pneumococcal clones of the Pneumococcal Molecular Epidemiology Network [12]. Band patterns were visually compared. Major PFGE patterns were defined as those including $>4$ isolates. At least one representative strain of each major PFGE pattern was analyzed by multilocus sequence typing (MLST) [13]. Allele numbers and sequence types were assigned using the MLST website [13]. Major lineages were named as clonal complexes (CC), with the serotype as superscript (e.g., CC $306^{1}$; clonal complex 306 of serotype 1 ). Susceptibility was determined by agar dilution [12]. Isolates with intermediate- or high-level resistance to oral/parenteral penicillin, cefotaxime (considering meningitis and non-meningitis breakpoints), erythromycin and levofloxacin determined using current Clinical and Laboratory Standards Institute (CLSI) breakpoints [14] were considered non-susceptible.

Vaccine types (VTs) were considered serotypes included in the PCV13 and serotype 6C based on previous data [15]. All other serotypes were considered non-vaccine types (NVTs).

Comparisons between proportions were performed using the $\chi^{2}$ test, Fisher's exact test and the Likelihood ratio test, as necessary. For quantitative variables, since data did not show normality in the Kolmogorov-Smirnoff test, the Kruskal-Wallis and MannWhitney tests were used as necessary. Statistical analyses were performed using the statistical program SPSS v.19 (SPSS Inc, Chicago IL).

Temporal trends of VTs (individually and overall), NVTs (overall, and individually for the most prevalent) and CCs within serotypes were calculated by linear/non-linear regression using time as the independent variable. The model showing the highest ratio $R^{2} /$ degrees of freedom was considered. Trends were considered significant when $p$-values were $\leq 0.05$.

\section{Results}

\subsection{Patients, episodes, serotypes and genotypes}

Of the 1107 IPDs included during the 5-year study period, 20 were excluded because they not met the eligibility criteria ( $\geq 24 \mathrm{~h}$ of hospitalization); thus, 1087 episodes were evaluable (see Supplementary material Table S1 for details on recruitment by hospital and study period). Strains from four episodes were not available for further studies. Among evaluable cases, 269 (24.7\%) occurred in 18-49-year-old patients, 273 (25.1\%) in 5064-year-old patients, $220(20.2 \%)$ in 64-74-year-old patients, and $325(29.9 \%)$ in patients older than 74 . The clinical presentations were: pneumonia (772, 71.0\%) (401 complicated and 371 uncomplicated), meningitis (122, 11.2\%), non-focal bacteremia (102, $9.4 \%)$, peritonitis $(34,3.1 \%)$ and other IPDs $(57,5.2 \%)$.

Supplementary data associated with this article can be found, in the online version, at https://doi.org/10.1016/j.vaccine.2018.10. 098.

Among the 1084 available isolates, the most common serotypes were: serotype 3 (12.7\%), 19A (8.5\%), 8 (7.7\%), 7F (6.3\%), 1 (4.2\%), $6 \mathrm{C}(4.2 \%), 11 \mathrm{~A}(4.2 \%), 22 \mathrm{~F}(4.2 \%)$ and $14(4.0 \%)$, accounting for $56 \%$ of the total isolates. VT (PCV13 +6C) accounted for 539 isolates (49.8\%). Table 1 shows the serotype distribution by study period. The percentage of VTs decreased over time $\left(R^{2}=0.812\right.$, $\beta=-0.901, p=0.037)$. Individually, serotypes $6 C \quad\left(R^{2}=0.821\right.$, $\beta=-0.906, p=0.034)$ and $7 \mathrm{~F}\left(\mathrm{R}^{2}=0.937, \beta=-0.964, p=0.007\right)$

Table 1

Annual distribution of serotypes causing IPDs in adults ( $\geq 18$ years) in Spain (August 2010-June 2015).

\begin{tabular}{|c|c|c|c|c|c|c|}
\hline & Isolates, $n(\%)$ & $2010 / 2011$ & $2011 / 2012$ & $2012 / 2013$ & $2013 / 2014$ & $2014 / 2015$ \\
\hline Serotypes & 1083 & 191 & 242 & 200 & 241 & 209 \\
\hline 1 & $46(4.2)$ & $12(6.3)$ & $8(3.3)$ & $13(6.5)$ & $12(5.0)$ & $1(0.5)$ \\
\hline 3 & 137 (12.7) & $22(11.5)$ & $27(11.2)$ & $27(13.5)$ & $28(11.6)$ & $33(15.8)$ \\
\hline 4 & $27(2.5)$ & $7(3.7)$ & $5(2.1)$ & $6(3.0)$ & $4(1.7)$ & $5(2.4)$ \\
\hline 5 & $1(0.1)$ & $0(0.0)$ & $0(0.0)$ & $1(0.5)$ & $0(0.0)$ & $0(0.0)$ \\
\hline $6 A$ & $8(0.7)$ & $3(1.6)$ & $2(0.8)$ & $1(0.5)$ & $2(0.8)$ & $0(0.0)$ \\
\hline 6B & $7(0.6)$ & $3(1.6)$ & $1(0.4)$ & $0(0.0)$ & $3(1.2)$ & $0(0.0)$ \\
\hline $6 C^{\mathrm{a}}$ & $45(4.2)$ & $11(5.8)$ & $11(4.5)$ & $10(5.0)$ & $7(2.9)$ & $6(2.9)$ \\
\hline $7 F^{a}$ & $68(6.3)$ & $16(8.4)$ & $20(8.3)$ & $12(6.0)$ & $13(5.4)$ & $7(3.3)$ \\
\hline $9 \mathrm{~V}$ & $19(1.8)$ & $2(1.0)$ & $7(2.9)$ & $2(1.0)$ & $5(2.1)$ & $3(1.4)$ \\
\hline 14 & $43(4.0)$ & $11(5.8)$ & $9(3.7)$ & $11(5.5)$ & $8(3.3)$ & $4(1.9)$ \\
\hline $18 C$ & $10(0.9)$ & $1(0.5)$ & $3(1.2)$ & $3(1.5)$ & $1(0.4)$ & $2(1.0)$ \\
\hline $19 A$ & $92(8.5)$ & $18(9.4)$ & $21(8.7)$ & $20(10.0)$ & $20(8.3)$ & $13(6.2)$ \\
\hline $19 F$ & $27(2.5)$ & $4(2.1)$ & $10(4.1)$ & $5(2.5)$ & $5(2.1)$ & $3(1.4)$ \\
\hline $23 F$ & $9(0.8)$ & $2(1.0)$ & $3(1.2)$ & $2(1.0)$ & $1(0.4)$ & $1(0.5)$ \\
\hline All PCV13 + 6C & $539(49.8)$ & $112(58.6)$ & $127(52.5)$ & $113(56.5)$ & $109(45.2)$ & $78(37.3)$ \\
\hline 8 & $83(7.7)$ & $12(6.3)$ & $17(7.0)$ & $9(4.5)$ & $17(7.1)$ & $28(13.4)$ \\
\hline $11 A^{a}$ & $46(4.2)$ & $0(0.0)$ & $10(4.1)$ & $10(5.0)$ & $15(6.2)$ & $11(5.3)$ \\
\hline $22 F$ & $46(4.2)$ & $7(3.7)$ & $11(4.5)$ & $5(2.5)$ & $9(3.7)$ & $14(6.7)$ \\
\hline $9 N$ & $38(3.5)$ & $6(3.1)$ & $4(1.7)$ & $4(2.0)$ & $10(4.1)$ & $14(6.7)$ \\
\hline $12 F$ & $35(3.2)$ & $2(1.0)$ & $10(4.1)$ & $3(1.5)$ & $9(3.7)$ & $11(5.3)$ \\
\hline $15 A$ & $32(3.0)$ & $6(3.1)$ & $9(3.7)$ & $9(4.5)$ & $6(2.5)$ & $2(1.0)$ \\
\hline $24 F$ & $29(2.7)$ & $6(3.1)$ & $5(2.1)$ & $8(4.0)$ & $5(2.1)$ & $5(2.4)$ \\
\hline $16 F$ & $28(2.6)$ & $7(3.7)$ & $4(1.7)$ & $6(3.0)$ & $5(2.1)$ & $6(2.9)$ \\
\hline $10 A$ & $22(2.0)$ & $4(2.1)$ & $4(1.7)$ & $0(0.0)$ & $8(3.3)$ & $6(2.9)$ \\
\hline All Non-PCV13 ${ }^{a}$ & $544(50.2)$ & $79(41.4)$ & $115(47.5)$ & $87(43.5)$ & $132(54.8)$ & $131(62.7)$ \\
\hline
\end{tabular}

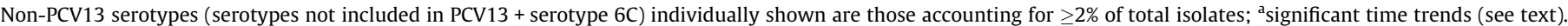

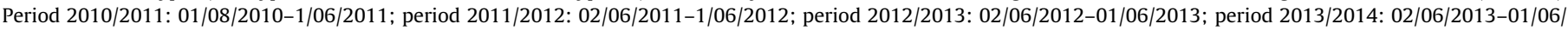
2014; period 2014/2015:02/06/2014-01/06/2015. 
decreased over time. NVTs significantly increased over the study period; individually, the percentage of serotype $11 \mathrm{~A}\left(\mathrm{R}^{2}=0.974\right.$, $\beta=3.543, p=0.027$ ) increased over time.

The percentage of VT isolates was higher in the area with low PCV13 pediatric uptake (Barcelona) than in the area with high coverage (Madrid) (53.9\% vs. 40.8\%, $p=0.001$ ), with differences for serotype $14(4.8 \% v s .1 .8 \%, p=0.048)$. Among NVTs, serotypes 8 and $16 \mathrm{~F}$ were more common in the region with high coverage $(16.9 \%$ vs. $3.9 \%, p<0.001$, and $4.9 \%$ vs. $0.8 \%, p=0.002$, respectively), while serotype $24 \mathrm{~F}$ was more common in the region with low uptake (5.3\% vs. $0.7 \%, p=0.001$ ).
Fig. 2 shows the major pneumococcal genotypes (CCs with $\geq 10$ isolates) by serotype. In general, there was an association between CCs and serotype, with the exception of $\mathrm{CC} 386^{6 \mathrm{C}, 6 \mathrm{~B}}$ CC156 ${ }^{14,11 \mathrm{~A}, 9 \mathrm{~V}, 19 \mathrm{~A}, 24 \mathrm{~F}}, \mathrm{CC} 230^{19 \mathrm{~A}, 24 \mathrm{~F}, 19 \mathrm{~F}}$ and CC63 $3^{8,15 \mathrm{~A}, 23 \mathrm{~B}, 19 \mathrm{~F}}$, which were associated with more than one serotype. Serotypes 3, 8 and 11A had two major CCs each. Although serotype 19A isolates were genetically diverse, two CCs (CC230 ${ }^{19 \mathrm{~A}}$ and CC $320^{19 \mathrm{~A}}$ ) accounted for $54.3 \%$ of $19 \mathrm{~A}$ isolates. The changes in the CCs over the study period are shown in Supplementary material Table S2.

Relationship of serotypes/genotypes with age, clinical presentation, and immune status

\section{PCV13 + 6C}

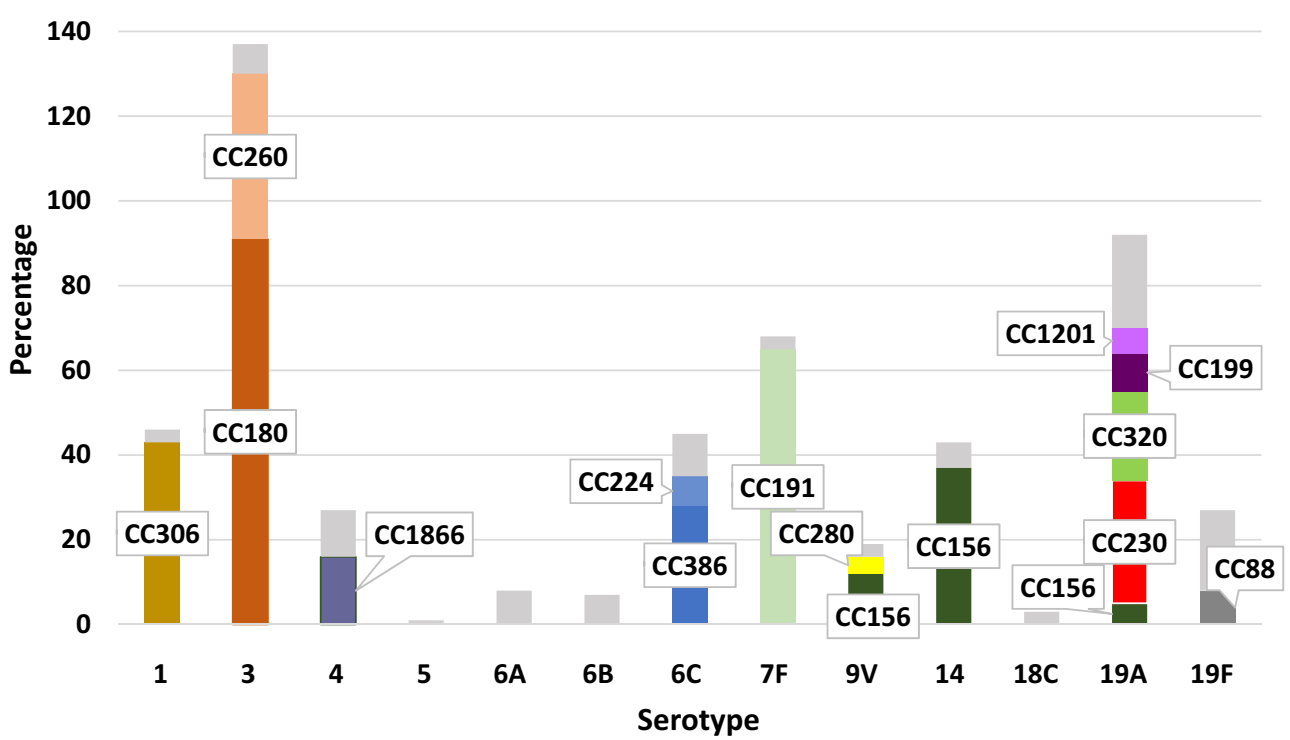

Grey color in bars represents $\mathrm{CC}(\mathrm{s})$ with $<10$ isolates

\section{Non-PCV13}

140

120

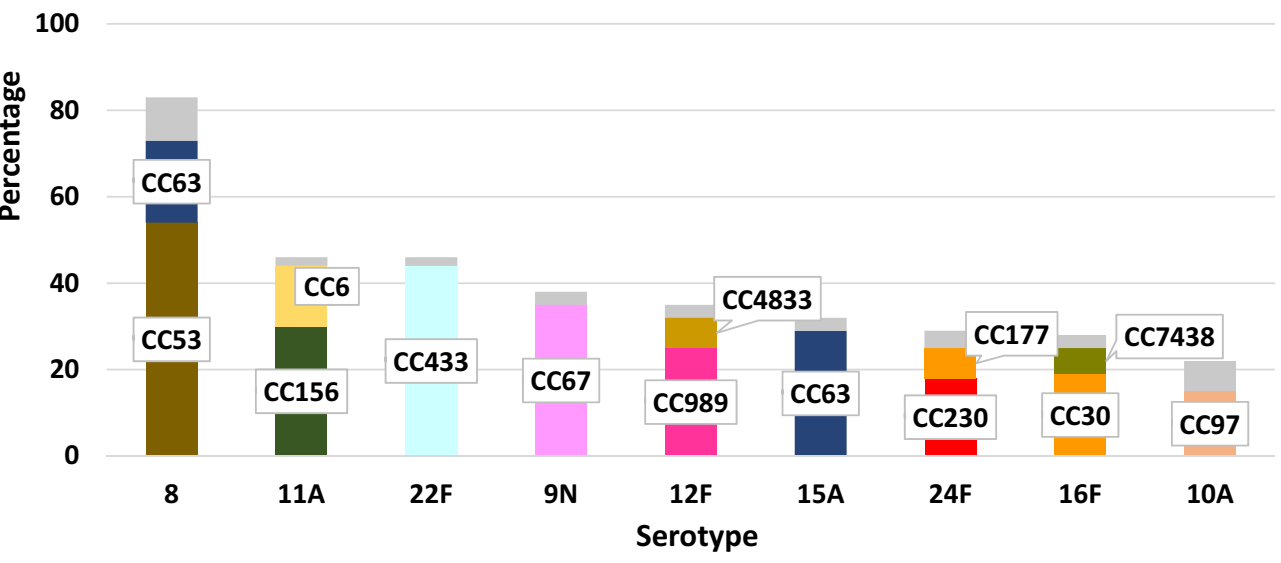

Grey color in bars represents $\mathrm{CC}(\mathrm{s})$ with $<10$ isolates

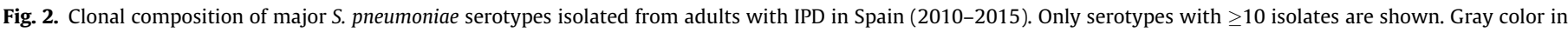
bars represents $\mathrm{CC}(\mathrm{s})$ with $<10$ isolates.

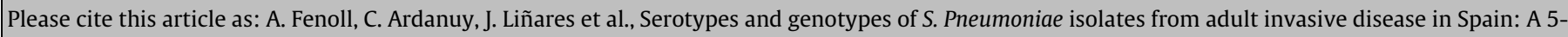
year prospective surveillance after pediatric PCV13 licensure. The ODIN study, Vaccine, https://doi.org/10.1016/j.vaccine.2018.10.098 
The most common serotypes among pneumonia isolates were: serotypes 3 (15.1\%), 8 (9.6\%), 19A (9.5\%) and 7F (7.7\%). VTs caused $53.9 \%$ ( 414 out of 768 ) of all pneumonia episodes, and 58.9\% (235 out of 399) of complicated pneumonia episodes (Supplementary material Table S3). Serotype 1 was most common among complicated $(7.0 \%)$ compared to uncomplicated pneumonia $(3.0 \%$, $p=0.01$ ). There were no differences regarding clonal composition of isolates causing complicated or uncomplicated pneumonia, except among serotype 3 CCs. There was an association between $\mathrm{CC} 180^{3}$ and complicated pneumonia (50 of 381 complicated pneumonia vs. 24 of 359 non-complicated pneumonia, $p=0.003$ ).

Among pneumonia isolates, $0.9 \%$ had penicillin minimum inhibitory concentrations (MICs) $\geq 4 \mu \mathrm{g} / \mathrm{mL}$ and $2.5 \%$ had cefotaxime MICs $\geq 2 \mu \mathrm{g} / \mathrm{mL}$ (non-meningeal breakpoints).

There were 122 meningitis episodes; serotype 3 (9.8\%), and serotypes $11 \mathrm{~A}$ and $19 \mathrm{~A}$ ( $7.4 \%$ each) were the most common (Supplementary Table S3). VT accounted for $43.4 \%$ of meningitis isolates. Using meningeal breakpoints, penicillin $(\geq 0.12 \mu \mathrm{g} / \mathrm{mL})$ and cefotaxime $(\geq 1 \mu \mathrm{g} / \mathrm{mL})$ resistance rates were $30.3 \%$ and $12.3 \%$, respectively.

Finally, serotypes 15A (10.8\%), 6C (9.8\%), 11A (6.9\%) and serotypes 3 and $9 \mathrm{~N}$ (5.9\% each) were the most common among nonfocal bacteremia isolates, with serotypes $11 \mathrm{~A}(14.7 \%), 6 \mathrm{C}(8.8 \%)$ and serotypes 7F, 14, 15B, 17F, 19A, 19F and $24 \mathrm{~F}$ (5.9\% each) the most common among peritonitis episodes. VTs accounted for $35.3 \%$ and $38.2 \%$ of non-focal bacteremia and peritonitis isolates, respectively.

By age group, the most common serotypes in young adults (<50 years) were serotypes 8 (10.4\%), 3 (10.1\%), 7F (8.6\%), 19A (6.7\%), 1 and 14 (5.6\% each), and 6C (5.2\%); VTs accounted for $53.4 \%$ of isolates. In patients $\geq 65$ years, the most common serotypes were 3 (13.5\%), 8 (5.5\%), 19A (7.9\%) and 22F (5.4\%), while VT-IPD represented $46.1 \%$ of all IPD (Supplementary Table S4).

Regarding immune status, 616 IPD episodes occurred in immunocompetent patients and 467 in immunocompromised patients (Supplementary Table S5). Forty-four different serotypes were found among immunocompetent patients, five of which accounted for more than $51 \%$ of episodes; the number of serotypes found among immunocompromised patients was similar (40), eight of which accounted for $50 \%$ of episodes. Serotypes $1(5.8 \%$ vs. $2.1 \%, p=0.003), 3$ (16.0\% vs. $8.1 \%, p<0.001)$, $7 \mathrm{~F}(7.8 \%$ vs. $4.3 \%$, $p=0.018)$ and $8(9.3 \%$ vs. $5.6 \%, p=0.024)$ were more frequent among immunocompetent patients, whereas serotypes $10 \mathrm{~A}(3.6 \%$ vs. $0.8 \%, p=0.002), 11 \mathrm{~A}(5.8 \%$ vs. $3.1 \%, p=0.043)$ and $15 \mathrm{~A}(5.1 \%$ vs. $1.3 \%, p \leq 0.001$ ) were more frequent among immunocompromised patients. In these two groups, VTs caused 54.7\% (337 out of 616) of IPD in immunocompetent patients and 43.3\% (202 out of 467) in immunocompromised patients.

\subsection{Antimicrobial non-susceptibility}

Table 2 shows non-susceptibility data. Overall, nonsusceptibility (MIC $\geq 0.12 \mathrm{mg} / \mathrm{L}$ ) was $25.9 \%$ [ $1.1 \%$ for parenteral MIC $\geq 4 \mathrm{mg} / \mathrm{L}$ )] to penicillin, $24.9 \%$ to erythromycin and $2.7 \%$ to levofloxacin. No differences in non-susceptibility were found between regions with high and low coverage, except for levofloxacin non-susceptibility, with a significantly higher percentage in the region with high coverage $(6.7 \% v s .1 .1 \%, p<0.001)$, associated with a higher percentage of serotype 8 .

Table 3 shows non-susceptibility rates for serotypes/CCs. Only 12 isolates were non-susceptible to parenteral penicillin (all $\mathrm{MIC}=4 \mathrm{mg} / \mathrm{L}$ ): seven serotype $19 \mathrm{~A}$ (all CC320), two 14 (both CC156), one 9V (CC156), one 6B and one 23F. According to nonmeningitis breakpoints for cefotaxime, 29 (2.7\%) isolates (28 $\mathrm{MIC}=2 \mathrm{mg} / \mathrm{L}$, one $\mathrm{MIC}=4 \mathrm{mg} / \mathrm{L}$ ) were non-susceptible: 16 serotype 19A (eleven CC320, three CC230, one CC156), nine serotype 14 (eight CC156), two 9V (CC156), one 6B and one 23F. Of the 270 isolates resistant to erythromycin, 239 (88.5\%) exhibited MIC $\geq 128 \mathrm{mg} / \mathrm{L}$. Serotypes with levofloxacin non-susceptibility $>10 \%$ were $9 \mathrm{~V}$ (21.1\%), 8 (19.3\%), 23F (11.1\%) and 9N (10.5\%). Eighteen out of $29(62.1 \%)$ isolates non-susceptible to levofloxacin belonged to CC63, of which 15 were serotype 8 and three were serotype $15 \mathrm{~A}$. Non-susceptibility by serotype within CCs was examined for three CCs expressing more than one capsule: CC63 ${ }^{8,15 A, 23 B, 19 F}, C C 156^{14,11 A, 9 V, 19 A, 24 F}$ and CC23019A,24F,19F. Within CC63, all isolates belonging to serotype 8 were susceptible to penicillin while all serotype $15 \mathrm{~A}$ were non-susceptible; levofloxacin non-susceptibility was $78.9 \%$ and $10.3 \%$ among isolates of serotypes 8 and 15A, respectively $(p<0.001)$. Within CC156, erythromycin non-susceptibility was $100 \%$ (19A), $25 \%(9 \mathrm{~V})$ and $\leq 8 \%$ (14 and $11 \mathrm{~A}$ ), while non-susceptibility to levofloxacin was $33.3 \%$ (9V), 2.7\% (14) and 0\% (11A and 19A).

\section{Discussion}

This multicenter study presents an overview of serotype and genotype distribution causing adult IPD in Spain, a country with regional differences in PCV pediatric uptake. Approximately half of IPDs in adults were caused by VTs, although the percentage decreased over the 5-year study period, reflecting herd protection by pediatric vaccination. Among NVTs, significant increases over time were found only for serotype $11 \mathrm{~A}$ (appearing in period 2011/2012). Regarding serotype $8 \mathrm{CC}^{8} 3^{8}$ decreased and CC53 ${ }^{8}$ increased. A recent Canadian study described genetic shifts within serotypes among isolates collected from children $\leq 5$ years old because of the impact of PCV13, and suggested selective

Table 2

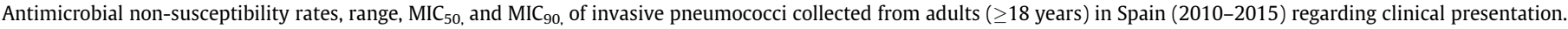

\begin{tabular}{|c|c|c|c|c|c|c|c|c|c|c|c|c|}
\hline & \multicolumn{4}{|c|}{ All isolates $(n=1083)$} & \multicolumn{4}{|c|}{ Meningitis $(n=122)$} & \multicolumn{4}{|c|}{ Pneumonia $(n=768)$} \\
\hline & Range & $\mathrm{MIC}_{50}$ & $\mathrm{MIC}_{90}$ & $\%$ & Range & $\mathrm{MIC}_{50}$ & $\mathrm{MIC}_{90}$ & $\%$ & Range & $\mathrm{MIC}_{50}$ & $\mathrm{MIC}_{90}$ & $\%$ \\
\hline Penicillin & $\leq 0.015-4$ & $\leq \mathbf{0 . 0 1 5}$ & 1 & & $\leq 0.015-4$ & $\leq \mathbf{0 . 0 1 5}$ & 1 & 30.3 & $\leq 0.015-4$ & $\leq \mathbf{0 . 0 1 5}$ & 1 & 0.9 \\
\hline$\geq \mathbf{0 . 1 2}$ & - & - & - & 25.9 & - & - & - & 30.3 & - & - & - & 22.9 \\
\hline$\overline{2}$ & - & - & - & 6.7 & - & - & - & 4.9 & - & - & - & 6.4 \\
\hline 4 & - & - & - & 1.1 & - & - & - & 2.5 & - & - & - & 0.9 \\
\hline Cefotaxime & $\leq 0.015-4$ & $\leq \mathbf{0 . 0 1 5}$ & 1 & & $\leq 0.015-4$ & $\leq \mathbf{0 . 0 1 5}$ & 1 & 12.3 & $\leq \mathbf{0 . 0 1 5 - 2}$ & $\leq \mathbf{0 . 0 1 5}$ & 1 & 2.5 \\
\hline 1 & - & - & - & 12.4 & - & - & - & 9.8 & - & - & - & 9.6 \\
\hline$\geq \mathbf{2}$ & - & - & - & 2.7 & - & - & - & 2.5 & - & - & - & 2.5 \\
\hline Erythromycin & $\leq \mathbf{0 . 1 2}-\geq 128$ & $\leq \mathbf{0 . 1 2}$ & $\geq 128$ & 24.9 & $\leq \mathbf{0 . 1 2}-\geq \mathbf{1 2 8}$ & $\leq \mathbf{0 . 1 2}$ & $\geq 128$ & 29.5 & $\leq \mathbf{0 . 1 2}-\geq 128$ & $\leq \mathbf{0 . 1 2}$ & $\geq 128$ & 21.0 \\
\hline Levofloxacin & $\leq 1-\geq 64$ & $\leq \mathbf{1}$ & $\leq \mathbf{1}$ & 2.7 & $\leq \mathbf{1 - 2}$ & $\leq \mathbf{1}$ & $\leq \mathbf{1}$ & 0.0 & $\leq 1-\geq 64$ & $\leq \mathbf{1}$ & $\leq \mathbf{1}$ & 2.9 \\
\hline
\end{tabular}

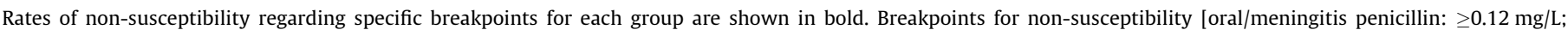
parenteral penicillin: $\geq 4 \mathrm{mg} / \mathrm{L}$; cefotaxime (meningitis): $\geq 1 \mathrm{mg} / \mathrm{L}$; cefotaxime (non-meningitis): $\geq 2 \mathrm{mg} / \mathrm{L} ;$ erythromycin: $\geq 0.5 \mathrm{mg} / \mathrm{L} ;$ levofloxacin: $\geq 4 \mathrm{mg} / \mathrm{L}$ ].

a Two isolates with MIC $=2 \mathrm{mg} / \mathrm{L}$. 
Table 3

Antimicrobial non-susceptibility rates for serotypes and related CCs of S. pneumoniae strains collected from adults ( $\geq 18$ years) with IPD in Spain (2010-2015).

\begin{tabular}{|c|c|c|c|c|c|}
\hline Serotype & Related $\mathrm{CCs}^{\mathrm{a}}$ & Penicillin & Cefotaxime & Erythromycin & Levofloxacin \\
\hline \multirow[t]{2}{*}{$6 C(n=45)$} & & $44.4 /-$ & $-1-$ & 66.7 & - \\
\hline & CC386 $(n=28)$ & $50.0 /-$ & $-1-$ & 100 & - \\
\hline \multirow[t]{3}{*}{$8(n=83)$} & & $-1-$ & $-1-$ & 22.9 & 19.3 \\
\hline & CC53 $(n=54)$ & $-1-$ & $-1-$ & - & 1.9 \\
\hline & $\operatorname{cc63}(n=19)$ & $-1-$ & $-1-$ & 100 & 78.9 \\
\hline \multirow[t]{2}{*}{$9 \mathrm{~N}(n=38)$} & & $5.3 /-$ & $-1-$ & 2.6 & - \\
\hline & CC67 $(n=35)$ & $2.9 /-$ & $-1-$ & 2.9 & - \\
\hline \multirow[t]{2}{*}{$9 \mathrm{~V}(n=19)$} & & $47.4 / 5.3$ & $36.8 / 10.5$ & 15.8 & 21.1 \\
\hline & $\operatorname{CC156}(n=12)$ & $75.0 / 8.3$ & $58.3 / 16.7$ & 25.0 & 33.3 \\
\hline \multirow[t]{2}{*}{$10 \mathrm{~A}(n=22)$} & & $-1-$ & $-1-$ & 31.8 & - \\
\hline & CC97 $(n=15)$ & $-1-$ & $-1-$ & 13.3 & - \\
\hline \multirow[t]{3}{*}{$11 \mathrm{~A}(n=46)$} & & $69.6 /-$ & $50.0 /-$ & 28.3 & - \\
\hline & $\operatorname{CC156}(n=30)$ & $100 /-$ & $76.7 /-$ & 6.7 & - \\
\hline & $\operatorname{CC62}(n=14)$ & $-1-$ & $-1-$ & 71.4 & - \\
\hline \multirow[t]{2}{*}{$14(n=43)$} & & $95.3 / 4.7$ & $83.7 / 20.9$ & 11.6 & 2.3 \\
\hline & CC156 $(n=37)$ & $100 / 5.4$ & $89.2 / 21.6$ & 8.1 & 2.7 \\
\hline \multirow[t]{2}{*}{$15 \mathrm{~A}(n=32)$} & & $90.6 /-$ & $9.4 /-$ & 90.6 & 9.4 \\
\hline & CC63 $(n=29)$ & $100 /-$ & $10.3 /-$ & 100 & 10.3 \\
\hline 15B $(n=20)$ & & $-1-$ & $-1-$ & 25.0 & - \\
\hline \multirow[t]{2}{*}{$16 \mathrm{~F}(n=28)$} & & $28.6 /-$ & $-1-$ & 17.9 & - \\
\hline & CC30 $(n=19)$ & $5.3 /-$ & $-1-$ & 26.3 & - \\
\hline \multirow[t]{3}{*}{$19 \mathrm{~A}(n=92)$} & & $68.5 / 7.6$ & $50.0 / 17.4$ & 60.9 & 1.1 \\
\hline & $\operatorname{CC230}(n=29)$ & $100 /-$ & $51.7 / 10.3$ & 65.5 & 3.4 \\
\hline & $\operatorname{CC} 320(n=21)$ & $100 / 33.3$ & $100 / 52.4$ & 95.2 & - \\
\hline $19 \mathrm{~F}(n=27)$ & & $59.3 /-$ & $33.3 /-$ & 70.4 & 3.7 \\
\hline \multirow{2}{*}{$23 \mathrm{~A}(n=20)$} & & $10.0 /-$ & $-1-$ & 60.0 & - \\
\hline & CC42 $(n=18)$ & $-1-$ & $-1-$ & 55.6 & - \\
\hline \multirow[t]{2}{*}{$23 \mathrm{~B}(n=21)$} & & $66.7 /-$ & $4.8 /-$ & 9.5 & - \\
\hline & $\operatorname{CC2372}(n=15)$ & $80.0 /-$ & $-1-$ & 6.7 & - \\
\hline \multirow[t]{2}{*}{$24 \mathrm{~F}(n=29)$} & & $69.0 /-$ & $-1-$ & 69.0 & - \\
\hline & $\operatorname{CC230}(n=18)$ & $100 /-$ & $-1-$ & 100 & - \\
\hline 35B $(n=18)$ & & $50.0 /-$ & $22.2 /-$ & 5.6 & - \\
\hline
\end{tabular}

Absence of non-susceptible isolates is shown by (-).

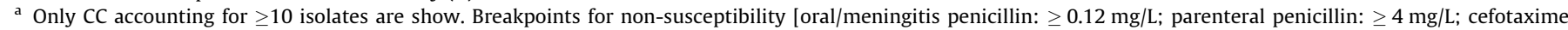
(meningitis): $\geq 1 \mathrm{mg} / \mathrm{L}$; cefotaxime (non-meningitis): $\geq 2 \mathrm{mg} / \mathrm{L}$; erythromycin: $\geq 0.5 \mathrm{mg} / \mathrm{L}$; levofloxacin: $\geq 4 \mathrm{mg} / \mathrm{L}]$.

advantages derived from genetic diversity, frequent recombination and drug resistance potential related to specific clones [16]. Based on this, not only the decline in VTs, but also the clonal changes in our study could be due to the herd effect after PCV13 introduction for children (only in those cases in which there was an association with serotypes included in the vaccine). However, explaining changes as derived from PCV13 uptake in children should be considered with caution, since PCV7/13 had not been universally administered for pediatric vaccination in all Spanish geographical areas, and isolates came from adults. In this sense, the analysis of isolates from a region with high vaccine uptake (PCV7/13 included in the pediatric immunization program; estimated uptake $>80 \%$ ) versus one region with low uptake [PCV7/13 not included by the period analyzed; estimated uptake $=55 \%$ [17] was performed to explore possible herd effects. Based on our findings, indirect effects can be inferred, since adult VT-IPDs were significantly less frequent in the area with high pediatric coverage. It has been reported that there is a decline in carriage of PCV13 serotypes in $\geq 50 \%$ of nonimmunized children only in communities where pediatric PCV13 coverage is $\geq 75 \%$, [18], which indirectly reduces the burden of VT-IPD. Finally, the genetic diversity or homogeneity of some serotypes and their variations could be related to changes in the clonal composition. For example, since nearly all serotype 7F strains belonged to CC191, the decrease in this serotype is associated with a decrease in the CC191. In contrast, the decrease in serotype 14 could not be related with a genotype, since CC156 isolates could express different capsular types including non-PCV13 serotypes.

In this study we have shown differences in the serotype composition in terms of clinical presentation, age and immune status, as has been previously described. Thus, VTs accounted for more than a half of pneumonia episodes. Since pneumonia is a major threat in the adult population, a PCV13 vaccination strategies could reduce the burden of this disease. In addition, some VTs (serotypes 1, 3, $19 \mathrm{~A}$ and $7 \mathrm{~F}$ ) were associated with complicated pneumonia as recently reported in Spain [19,20]. Notably, among pneumonia episodes due to serotype 3 those caused by $\mathrm{CC} 180^{3}$ were more frequent among complicated pneumonia suggesting that other factors beside capsule are also important in the pathogenesis of infection. Moreover, with respect to immune status non-invasive serotypes $(11 \mathrm{~A}, 9 \mathrm{~N}, 15 \mathrm{~A}, 10 \mathrm{~A})$ were more frequent among immunosuppressed patients, suggesting that the impaired immune system could facilitate the invasiveness of these serotypes [21]. Furthermore, invasive serotypes were more common among immunocompetent patients $(1,3,7 \mathrm{~F}, 8)$ highlighting the major role of the capsular polysaccharide in the invasiveness.

Previous studies have suggested that adults with high-risk conditions may not benefit from indirect protection as much as immunocompetent patients [10,22]. Nevertheless, VTs caused $43.3 \%$ IPD in immunocompromised patients that could take advantage of PCV13 vaccination until herd protection from pediatric PCV13 is fully established [23].However, considering our data from the area, where the proportion of VT-IPDs remains high despite $>80 \%$ pediatric coverage, a better strategy to reduce the burden of adult IPD would be to offer a specific PCV13 vaccination program not only to high-risk adults (as currently done in Spain), but to all persons aged $\geq 60$ years old. Hence, ACIP recommends that PCV13 is routinely administered to all adults aged $\geq 65$ years [24]. Specific vaccination programs in adults have been suggested, since serotypes causing IPDs in adults are rarely found in the nasopharynx of children [25].

In line with other studies performed in Spain [11,26], our results shows that penicillin/erythromycin non-susceptibility is linked to serotypes $15 \mathrm{~A}, 19 \mathrm{~A}, 6 \mathrm{~B}, 19 \mathrm{~F}$ and $24 \mathrm{~F}$, while levofloxacin 
non-susceptibility was mainly linked to serotype 8 [27-29]. The relationship between serotype 8 and levofloxacin-resistance was previously described associated with the spread of a recombinant clone 8-ST63 (related to clone Sweden15A-ST63) initially confined in Madrid area and associated with HIV patients [27,29], that expanded to other regions [27]. Although the main reservoir of pneumococci are children, isolates of serotype 8 are rarely found, neither as colonizers or agents of invasive disease [30]. Notably, since fluoroquinolones are not used in children, the quinoloneresistance in $\mathrm{CC}^{\circ} 3^{8}$ represents a problem strictly in adults. Importantly, in our study we observed a decrease of CC63 within serotype 8 , with a significant increase in $\mathrm{CC}^{8} 3^{8}$ (susceptible to penicillin, erythromycin and levofloxacin). Finally, the continuing presence of CCs strongly linked to high beta-lactam resistance, such as CC320 (serotype 19A) and CC156 (serotypes 14, 9V and $11 \mathrm{~A}$ ), is also a concern. However, the percentage of serotype 14 was higher among regions with lower vaccination coverage in children (data not shown) [17]. This reinforces the need for high vaccination coverage in children to protect the main population affected, and through the herd effect to protect subjects not targeted by vaccination strategies.

The results of the present surveillance study showed that, although the percentage of VTs as a cause of IPDs in adults in Spain is decreasing, it remains high, even in areas where PCV7/13 is included in the pediatric immunization program. Moreover, this study shows differences in the serotype distribution as regards clinical presentation, age or immune status highlighting the role of capsular type in the pathogenesis of pneumococcal infections. The main limitation of this study is that it is not a populationbased study which hampered to know the real impact of children vaccination among the burden of adult IPD. However, our study analyzes a large pneumococcal series causing IPD in adults allowing us to analyze the serotype composition and vaccine coverage of IPD in different populations targeted by the current 13-valent conjugate vaccine.

\section{Potential conflicts of interest}

This study was sponsored by Pfizer S.L.U., Madrid, Spain. Part of this study was presented at the 26th European Congress of Clinical Microbiology and Infectious Diseases (ECCMID), Amsterdam, Netherlands (April 9-12, 2016) and at the 10th International Symposium on Pneumococci and Pneumococcal Diseases (ISPPD), Glasgow, UK (June 26-30, 2016).

A.F., C.A. and J.L. report grants to their Institutions from Pfizer S. L.U., Madrid, Spain, for this study, and support for travel to meetings for the study or other purposes from Pfizer S.L.U. during the conduct of the study. E.C., F.M., A.F., M.R-M., J-L.L-H., B.P., A-I.A., and B.B. report grants to their Institutions from Pfizer S.L.U., Madrid, Spain, for this study. C.M. and I.C. are employees of Pfizer S.L.U., Madrid, Spain.

Other members of the ODIN Study Group are:

R. Pallarés, I. Grau, L. Calatayud, M. Cubero (Hospital Universitario de Bellvitge-IDIBELL, Barcelona); A. Torres, E. Polverino, C. Cillóniz, R. Amaro (Hospital Clinic i Provincial, Barcelona); L. Molinos, A. Pando (Hospital Universitario Central de Asturias, Oviedo); J. Aspa, B. Buendía, O. Rajas, C. de las Cuevas (Hospital Universitario La Princesa, Madrid); E. Bouza, M. Rodríguez-Créixems, A. Fernández, A. Burillo (Hospital General Universitario Gregorio Marañón, Madrid); P. Llinares, M.C. Zúñiga, D. Sousa, L. Castelo (Complejo Hospitalario Universitario A Coruña, A Coruña); J.L. De la Cruz, I. De Toro, S. Rincón, L. Luque (Hospital Regional Universitario Carlos Haya, Málaga); I. Alfageme, N. Reyes (Hospital Nuestra Señora de Valme, Sevilla); M. Salavert, A. Gil, S. Cuellar (Hospital Universitario y Politécnico La Fe, Valencia); C. Balseiro, M. Del Amo, A.
García, Juan Sáez (Pfizer S.L.U., Madrid); and M.J. Giménez, L. Aguilar (PRISM-AG, Madrid).

\section{References}

[1] Hausdorff WP, Feikin DR, Klugman KP. Epidemiological differences among pneumococcal serotypes. Lancet Infect Dis 2005;5:83-93.

[2] Geno KA, Gilbert GL, Song JY, Skovsted IC, Klugman KP, Jones C, et al. Pneumococcal capsules and their types: past, present, and future. Clin Microbiol Rev 2015;28:871-99.

[3] Fitzwater SP, Chandran A, Santosham M, Johnson HL. The worldwide impact of the seven-valent pneumococcal conjugate vaccine. Pediatr Infect Dis J 2012;31:501-8.

[4] Lepoutre A, Varon E, Georges S, Dorleans F, Janoir C, Gutmann L, et al. Impact of the pneumococcal conjugate vaccines on invasive pneumococcal disease in France, 2001-2012. Vaccine 2015;33:359-66.

[5] Picazo J, Ruiz-Contreras J, Casado-Flores J, Giangaspro E, Del Castillo F, Hernandez-Sampelayo T, et al. Relationship between serotypes, age, and clinical presentation of invasive pneumococcal disease in Madrid, Spain, after introduction of the 7-valent pneumococcal conjugate vaccine into the vaccination calendar. Clin Vacc Immunol CVI 2011;18:89-94.

[6] Waight PA, Andrews NJ, Ladhani SN, Sheppard CL, Slack MP, Miller E. Effect of the 13-valent pneumococcal conjugate vaccine on invasive pneumococcal disease in England and Wales 4 years after its introduction: an observational cohort study. Lancet Infect Dis 2015;15:535-43.

[7] Grau I, Ardanuy C, Cubero M, Benitez MA, Linares J, Pallares R. Declining mortality from adult pneumococcal infections linked to children's vaccination. J Infect 2016;72:439-49.

[8] Pilishvili T, Lexau C, Farley MM, Hadler J, Harrison LH, Bennett NM, et al. Sustained reductions in invasive pneumococcal disease in the era of conjugate vaccine. J Infect Dis 2010;201:32-41.

[9] Pletz MW, Ewig S, Rohde G, Schuette H, Rupp J, Welte T, et al. Impact of pneumococcal vaccination in children on serotype distribution in adult community-acquired pneumonia using the serotype-specific multiplex urinary antigen detection assay. Vaccine 2016;34:2342-8.

[10] Updated recommendations for prevention of invasive pneumococcal disease among adults using the 23-valent pneumococcal polysaccharide vaccine (PPSV23). MMWR Morbid Mortal Week Rep 2010;59:1102-6.

[11] Fenoll A, Granizo JJ, Gimenez MJ, Yuste J, Aguilar L. Secular trends (1990-2013) in serotypes and associated non-susceptibility of S. pneumoniae isolates causing invasive disease in the pre-/post-era of pneumococcal conjugate vaccines in Spanish regions without universal paediatric pneumococcal vaccination. Vaccine 2015:33:5691-9.

[12] McGee L, McDougal L, Zhou J, Spratt BG, Tenover FC, George R, et al. Nomenclature of major antimicrobial-resistant clones of Streptococcus pneumoniae defined by the pneumococcal molecular epidemiology network. J Clin Microbiol 2001;39:2565-71.

[13] Enright MC, Spratt BG. A multilocus sequence typing scheme for Streptococcus pneumoniae: identification of clones associated with serious invasive disease. Microbiology 1998;144:3049-60.

[14] Clinical Laboratory Standasds Institute (CLSI). Performance standards for antimicrobial susceptibility testing: nineteenth informational supplement M100-S19. Wayne (PA): Clinical Laboratory Standards Institute; 2009.

[15] Cohen R, Levy C, Bingen E, Koskas M, Nave I, Varon E. Impact of 13-valent pneumococcal conjugate vaccine on pneumococcal nasopharyngeal carriage in children with acute otitis media. Pediatr Infect Dis J 2012;31:297-301.

[16] Deng X, Arya G, Memari N, Mackenzie R, MacMullin G, Low DE, et al. Genetic analysis of invasive pneumococcal isolates from children in Ontario, Canada, 2007-2012. Pediatr Infect Dis J 2015;34:594-8.

[17] Del Amo E, Esteva C, Hernandez-Bou S, Galles C, Navarro M, Sauca G, et al. Serotypes and clonal diversity of streptococcus pneumoniae causing invasive disease in the era of PCV13 in Catalonia, Spain. PloS one 2016;11:e0151125.

[18] Loughlin AM, Hsu K, Silverio AL, Marchant CD, Pelton SI. Direct and indirect effects of PCV13 on nasopharyngeal carriage of PCV13 unique pneumococcal serotypes in Massachusetts' children. Pediatr Infect Dis J 2014;33:504-10.

[19] Sanz-Herrero F, Gimeno-Cardona C, Tormo-Palop N, Fernandez-Fabrellas E, Briones ML, Cervera-Juan A, et al. The potential role of 13-valent pneumococcal conjugate vaccine in preventing respiratory complications in bacteraemic pneumococcal community-acquired pneumonia. Vaccine 2016;34:1847-52.

[20] Tagarro A, Benito A, Sanchez A, Aznar E, Otheo E, Sanz-Rosa D. Bacteremic pneumonia before and after withdrawal of 13-valent pneumococcal conjugate vaccine from a public vaccination program in Spain: a case-control study. J Pediatr 2016;171(111-5):e1-3.

[21] Brueggemann AB, Griffiths DT, Meats E, Peto T, Crook DW, Spratt BG. Clonal relationships between invasive and carriage Streptococcus pneumoniae and serotype- and clone-specific differences in invasive disease potential. J Infect Dis 2003:187:1424-32.

[22] Muhammad RD, Oza-Frank R, Zell E, Link-Gelles R, Narayan KM, Schaffner W, et al. Epidemiology of invasive pneumococcal disease among high-risk adults since the introduction of pneumococcal conjugate vaccine for children. Clin Infect Dis Off Publ Infect Dis Soc Am 2013;56:e59-67.

[23] Shigayeva A, Rudnick W, Green K, Chen DK, Demczuk W, Gold WL, et al. Invasive pneumococcal disease among immunocompromised persons: 
implications for vaccination programs. Clin Infect Dis Off Publ Infect Dis Soc Am 2016;62:139-47.

[24] Tomczyk S, Bennett NM, Stoecker C, Gierke R, Moore MR, Whitney CG, et al. Use of 13-valent pneumococcal conjugate vaccine and 23-valent pneumococcal polysaccharide vaccine among adults aged $>/=65$ years: recommendations of the Advisory Committee on Immunization Practices (ACIP). MMWR Morb Mortal Wkly Rep 2014;63:822-5.

[25] Azzari C, Cortimiglia M, Nieddu F, Moriondo M, Indolfi G, Mattei R, et al. Pneumococcal serotype distribution in adults with invasive disease and in carrier children in Italy: should we expect herd protection of adults through infants' vaccination? Human Vacc Immunotherap 2016;12:344-50.

[26] Linares J, Ardanuy C, Pallares R, Fenoll A. Changes in antimicrobial resistance, serotypes and genotypes in Streptococcus pneumoniae over a 30-year period. Clin Microbiol Infect Off Publ Eur Soc Clin Microbiol Infect Dis 2010;16:402-10.
[27] Ardanuy C, de la Campa AG, Garcia E, Fenoll A, Calatayud L, Cercenado E, et al Spread of Streptococcus pneumoniae serotype 8-ST63 multidrug-resistant recombinant Clone, Spain. Emerg Infect Dis 2014;20:1848-56.

[28] Domenech A, Tirado-Velez JM, Fenoll A, Ardanuy C, Yuste J, Linares J, et al Fluoroquinolone-resistant pneumococci: dynamics of serotypes and clones in Spain in 2012 compared with those from 2002 and 2006. Antimicrob Agents Chemother 2014;58:2393-9.

[29] Sanz JC, Cercenado E, Marin M, Ramos B, Ardanuy C, Rodriguez-Avial I, et al Multidrug-resistant pneumococci (serotype 8) causing invasive disease in HIV + patients. Clin Microbiol Infect Off Publ Eur Soc Clin Microbiol Infect Dis 2011;17:1094-8.

[30] Sleeman KL, Griffiths D, Shackley F, Diggle L, Gupta S, Maiden MC, et al. Capsular serotype-specific attack rates and duration of carriage of Streptococcus pneumoniae in a population of children. J Infect Dis 2006;194:682-8. 